\title{
Joachim Perels \\ Neue Studien über die juristische Struktur des Dritten Reiches
}

\section{$\mathrm{Zu}$ Arbeiten von Horst Dreier}

Die Erforschung der juristischen Lehre der NS-Diktatur war lange Jahre in der Rechtswissenschaft der Bundesrepublik ein Nicht-Thema. Die großen, in der Emigration auf Englisch veröffentlichten, leicht zugänglichen Analysen der juristischen Struktur des Dritten Reiches von sozialdemokratischen Juristen wie Ernst Fraenkel, Franz L. Neumann und Otto Kirchheimer ${ }^{\mathrm{I}}$ wurden bis Mitte der sechziger Jahre von der universitären Rechtslehre - bis auf wenige Ausnahmen - nicht zur Kenntnis genommen. Das hatte einen einfachen Grund. Die Arbeiten der Emigration bezogen sich wesentlich auf die autoritären und rassistischen Konstruktionen jener Professoren des Nationalsozialismus, die in der Bundesrepublik unter den Bedingungen einer rechtsstaatlich-demokratischen Verfassung eine führende Rolle spielten. Um ihren wissenschaftlichen Anspruch zu sichern, belegten sie ihre affirmative Rolle in der Diktatur mit einem umfassenden Reflexionsverbot.

Angestoßen durch die bahnbrechende Untersuchung von Bernd Rüthers von I 968 über die »Unbegrenzte Auslegung «² in der NS-Diktatur hat sich seit den siebziger Jahren eine umfangreiche kritische Forschung sowohl zur Rolle der universitären Jurisprudenz wie der Justiz entwickelt, die in viel besuchten Ausstellungen des Bundesjustizministeriums und des Niedersächsischen Justizministeriums einen konzentrierten Niederschlag gefunden hat. 3

Gleichwohl wirkte, insbesondere im öffentlichen Recht, das überkommene Schweigen über die Rechtslehre des Hitler-Regimes in bestimmter Beziehung fort. Als Michael Stolleis I 993 einen Aufsatz über die regimetreue Rolle des später führenden Grundgesetzkommentators, Theodor Maunz, veröffentlichte und hierfür von führenden Staatsrechtlern angegriffen wurde, war es nicht möglich, die Frage der Ausformung und Legitimierung der juristischen NS-Doktrin auf einer Tagung der Staatsrechtslehrer zum Gegenstand wissenschaftlicher Analyse und Diskussion zu machen. Erst 55 Jahre nach dem Zerbrechen der NS-Herrschaft sah sich die Vereinigung Deutscher Staatsrechtslehrer dazu in der Lage. Der Referent, Horst Dreier, Jahrgang 1954, Professor für Öffentliches Recht und Rechtsphilosophie an der Universität Würzburg, hatte schon lebensgeschichtlich keinerlei Beziehung zum NS-System. Sein Vortrag über »Die deutsche Staatsrechtslehre in der Zeit des Nationalsozialismus 4 und einige seiner anderen Arbeiten wie »Wirtschaftsraum - Großraum - Lebens-

I Ernst Fraenkel, Der Doppelstaat (194I), Frankfurt/Main 1974; Franz L. Neumann, Behemoth. Struktur und Praxis des Nationalsozialismus (1942/44), Köln 1977; Otto Kirchheimer, Die Rechtsordnung des Nationalsozialismus (194I), Kritische Justiz H. 4/r97I, S. 356 ff.

2 Bernd Rüthers, Die unbegrenzte Auslegung. Zum Wandel der Privatrechtsordnung im Nationalsozialismus (1968), Frankfurt/Main I973.

3 Im Namen des Deutschen Volkes. Justiz im Nationalsozialismus. Katalog zur Ausstellung des Bundesministers der Justiz, Köln I989; Justiz im Nationalsozialismus. Über Verbrechen im Namen des Deutschen Volkes. Beiträge und Katalog zur Ausstellung. Redaktion: Susanne Benzler, Monika Gödecke, BadenBaden 2002.

4 Horst Dreier, Die deutsche Staatsrechtslehre in der Zeit des Nationalsozialismus, Veröffentlichungen der Vereinigung der Deutschen Staatsrechtslehrer, 60, Berlin 200I, S. Io ff. (Dreier I). Vgl. auch Michael Stolleis, Theodor Maunz - Ein Staatsrechtslehrerleben, Kritische Justiz H. 4/1993, S. 393 ff. 
raum «5 und »Rechtszerfall und Kontinuität $\aleph^{6}$ zu juristischen Problemen der

NS-Herrschaft sind ein Zeichen dafür, dass mittlerweile der Nationalsozialismus auch innerhalb der rechtswissenschaftlichen Zunft umfassend kritisch analysiert wird. Die Wahrnehmungsabwehr der Generation der einstigen regimetreuen Juristen ist nun vollständig überwunden. Dreier legt die innere Logik der nationalsozialistischen Staatsrechtslehre, ihre Stoßrichtung und ihre Struktur systematisch bloß. Dabei reduzieren sich Positionen von diktaturtreuen Juristen nicht auf individuelle Verfehlungen, sie werden als legitimatorischer Teil des gesamten Herrschaftssystems kenntlich. Die juristischen Formen des NS-Regimes werden in ausgreifenden, durchweg genau belegten Kategorisierungen von Dreier an der Negation der wesentlichen Grundrechte, der rechtstechnischen Formierung des Antisemitismus und der machtzentralisierenden Aufhebung des Föderalismus kenntlich gemacht. Auf diese drei Funktionsbereiche sind die zentralen juristischen Herrschaftstechniken der Diktatur bezogen, die vor allem in den Arbeiten von Ernst Forsthoff, Ernst Rudolf Huber, Hans Peter Ipsen und Ulrich Scheuner - allesamt später führende Verfassungsrechtler der Bundesrepublik - auf den zeitgenössischen ideologischen Begriff gebracht werden. Sie sprechen die juristische Sprache des Regimes. Bei Forsthoff heißt es: »Grundrechte gehören der Geschichte an. «7 Hans-Peter Ipsen bringt in den Fußnoten bei Stahl, Laband und Nawiasky »hinter den Autorennamen in Klammern den Zusatz >Jude an. $^{8}$ Für die rechtsstaatsfeindliche Systemstruktur des Nationalsozialismus findet Huber prägnante Formeln: »Die Führergewalt ist umfassend und total ... Die Führergewalt ist nicht durch Sicherungen und Kontrollen, durch autonome Schutzbereiche und wohlerworbene Einzelrechte gehemmt, sondern sie ist frei und unabhängig, ausschließlich und unbeschränkt. $₫ 9$

Die Untersuchung Dreiers zeichnet sich dadurch aus, dass sie die in das juristische Denken transformierten Elemente des Dritten Reiches nicht unmittelbar moralisch-politisch wertend kritisiert (wenngleich dies selbstredend keinesfalls illegitim wäre), sondern in ihrer Bedeutung für die Formierung des neuen Regimes sichtbar werden lässt. Dieser rechtstheoretische Blick erfasst die Zerstörung eines rechtsstaatlichen liberalen Rechtssystems durch die gegenaufklärerische Rechtslehre in grundlegender Weise.

Allerdings wird die am Ende der Untersuchung in einem Satz zusammengedrängte Behauptung, dass es keine »substanziellen Fortwirkungen nationalsozialistischer Denkweisen in der Staatsrechtslehre gäbe und dass sich »etatistische Positionen im normalen Spektrum funktionierender Verfassungsstaaten $«$ hielten, ${ }^{1 \circ}$ nicht begründet. Die vielfache Affirmation der nationalsozialistischen Rechtsordnung, die sich in der Negation der diktaturkritischen Judikatur des Bundesverfassungsgerichts durch die erdrückende Mehrheit der Staatsrechtslehrer in den fünfziger Jahren niederschlägt, in der Rechtsprechung, die die Verfolgung der Widerstandskämpfer, der Juden, der Zivilbevölkerung und der Roma und Sinti für rechtmäßig erklärt, verweist auf die fortdauernde

\footnotetext{
5 Horst Dreier, Wirtschaftsraum - Großraum - Lebensraum, in: ders. u.a. (Hrsg.), Raum und Recht, Festschrift 600 Jahre Würzburger Juristenfakultät, Berlin 2002, S. 47 ff. (Dreier II).

6 Horst Dreier, Rechtszerfall und Kontinuität, Der Staat, H. 2/2004, S. 235 (Dreier III).

7 Dreier I, S. 25 , Anm. 77.

8 Dreier I, S. 32, Anm. I09.

9 Dreier I, S. 49 f.

Io Dreier I, S. 69.
} 
Hypothek der Rechtsdoktrin des Dritten Reichs. ${ }^{\text {I }}$ Auch die Aushöhlung der politischen Freiheitsrechte wie des Demonstrations- und Streikrechts und der Reduktion der Gestaltungsfreiheit des demokratischen Gesetzgebers auf die Reproduktion der bürgerlichen Gesellschaft ist bedingt durch die Interpretationsmacht der früheren juristischen Stützen der Diktatur. ${ }^{\text {I2 }}$

Das Ende des Vortrags von Dreier enthält dadurch eine besondere Pointe, dass er auf das Erkenntnisniveau der Emigrationsarbeiten von Fraenkel und Neumann hinweist, um an deren Kerngedanken mit dem letzten Satz seiner Untersuchung anzuschließen: »I933 ersehnten sich viele Bürger und weite Teile der Staatsrechtslehre einen starken, autoritären Staat - den Leviathan; doch der Oberwolf bescherte ihnen den Behemoth - den Un-Staat. «I3

In einem späteren Beitrag Dreiers steht das Verhältnis von Staatsrecht und Wirtschaftssystem im Nationalsozialismus im Mittelpunkt. Dieser Frage, die in der Sozial- und Geschichtswissenschaft schon vielfach, auch kontrovers, behandelt worden ist, gibt Dreier durch die eingehende Rezeption der zeitgenössischen juristischen Literatur und durch die Heranziehung historischer Forschungen eine besondere Wendung. Er zeigt, dass das Wirtschaftssystem des Dritten Reiches bei allen staatlichen Eingriffen und der Diskriminierungen der Juden prinzipiell an den Regeln des Privatrechtsverkehrs, selbst in der Kriegsökonomie, festhielt. Dreier resümiert: »Das Institut des Privateigentums (blieb) ebenso unangetastet wie die Struktur des Bürgerlichen Gesetzbuchs. Die ,Arisierungen' von Betrieben wiederum bedeuteten Personenaustausch, nicht Systemveränderung ... So beruhte die wirtschaftliche Produktion ... in allerdings stets enger werdenden Grenzen auf privatwirtschaftlicher Unternehmerinitiative; von allgemeiner Missachtung des Erfolgs individuellen Gewinnstrebens ... konnte nicht die Rede sein. «14

Die Entschlüsselung der juristischen Funktionsweise des NS-Systems enthält jedoch einige argumentative Bruchstellen, die im Widerspruch zu Dreiers eigener kritischer Vorgehensweise stehen und die die Distanz zur NS-Rechtsdoktrin zum Teil relativieren.

Bei der Erörterung der Bedeutung des Antisemitismus in der Staatsrechtslehre des Dritten Reiches ist davon die Rede, »... dass es einen erheblichen Unterschied « ausmache, ob in den Texten »allgemein gehaltene Formeln von der Art und Rassegebundenheit des Rechts ... begegnen oder mit - zum Teil personenbezogenen - antijüdischen Äußerungen gearbeitet wird.«Is Diese Unterscheidung ist fragwürdig. Auch wenn sie im Blick auf bestimmte Formulierungen, etwa von Ulrich Scheuner und Carl Schmitt, zutrifft, ${ }^{16}$ bleibt unterbelichtet, was die beiden Antisemitismen miteinander verbindet: die den Gleichheitssatz negierende Aussonderung der Juden mit juristischen Mitteln. Die Differenz zwischen einem so genannten Radau-Antisemitismus und der so genannten kühl-admini-

I I Vgl. Joachim Perels, Die Nachwirkungen der NS-Diktatur im demokratischen Rechtsstaat, in: ders., Das juristische Erbe des »Dritten Reiches«. Beschädigungen der demokratischen Rechtsordnung, Frankfurt/ Main 1999, S. I I ff.; Joachim Perels, Das Grundgesetz zwischen historischen Erfahrungen und tradierter Interpretationsmacht, in: ders., Entsorgung der NS-Herrschaft?, Hannover 2004, S. I I 9 ff.

I 2 Joachim Perels, Wolfgang Abendroth und die Rechtslehre der Ära Adenauer. Verteidigung des Grundgesetzes gegen konservative Interpretationsmacht, in: Zukunft der Demokratie in Deutschland, hrsgg. von Andrea Gourd/ Thomas Nötzel, Marburg 200 I, S. I3 I ff.

I 3 Dreier I, S. 67.

I4 Dreier II, S. 259.

Is Dreier I, S. 29, Anm. 2.

I6 Ulrich Scheuner, Der Gleichheitsgedanke in der völkischen Verfassungsordnung, Zeitschrift für die gesamte Staatswissenschaft, Bd. 99, I939, S. 245 ff.; Carl Schmitt, Die deutsche Rechtswissenschaft im Kampf gegen den jüdischen Geist, Deutsche Juristen-Zeitung H. 20/I936, S. I I $57 \mathrm{ff.}$ 
strativen Judenfeindschaft, die gerade von den Spitzen der SS gefordert wurde, ${ }^{17}$ ist nur ein Unterschied in der Methode, nicht im Ziel, die Juden aus dem gesellschaftlichen und staatlichen Leben auszustoßen.

Bei Ernst Rudolf Huber, ${ }^{18}$ den Dreier als Vertreter einer nicht personalpolitisch gerichteten, allgemeinen juristischen Rassendoktrin charakterisiert, wird jedoch die gesamte Kasuistik der Erniedrigung und Entrechtung der Juden - bis hin zu dem staatlich erzwungenen Verzicht auf Entschädigungszahlungen nach der Reichspogromnacht vom 9. November 1938 - als geltendes Recht konkret programmiert. ${ }^{19}$

In dem Aufsatz zur rechtlichen Organisationsform des Raums findet sich eine Interpretation der zeitgenössischen Völkerrechtstheorie von Carl Schmitt von I939, die zum Widerspruch herausfordert. Dreier vertritt die These, dass Schmitts auf dem Hintergrund der Expansionsziele des NS-Regimes entwickelte Doktrin, der zufolge an die Stelle der rechtlich geschützten Souveränität der Staaten ein insbesondere von Deutschland dominierter Großraum trete, der den Einfluss auf zuvor unabhängige Staaten sichere, ${ }^{20}$ ihre sachliche Berechtigung besitze. Schmitts Ausführungen besäßen eine »subjektive Plausibilität «, ja einen »innovativen und vorwärts weisenden Gehalt. «" ${ }^{21}$ Sein Verdienst bestehe darin, dass "zentrale Elemente der klassischen Völkerrechtslehre (Gleichheit der Staaten, Souveränität etc.) auf den Prüfstand gestellt werden, um neue begriffliche Instrumente zur Erfassung, wenn auch nicht zwingend Hegung, notwendig «22 zu machen. Mit dieser Bemerkung wird verkannt, dass Schmitts Lehre, die sich ausdrücklich auf die Politik des Reichskanzlers Hitler berief, ${ }^{23}$ nicht eine wertfreie Erkenntnis einer neuen völkerrechtlichen Lage zum Inhalt hatte. Sie war strategisch bedingt, denn sie versah die imperialistische Politik des NS-Regimes mit einer rechtlich drapierten Ideologie. Sie zielte ausdrücklich auf die Niederreißung der Prinzipien des Völkerbundes, die den Interessen Nazi-Deutschlands entgegenstanden. Die Wahrnehmung des empirisch feststellbaren Unterschieds des Gewichts von Großmächten und kleineren Mächten, die Dreier als Erkenntnisleistung Schmitts wertet, ist, wenn Völkerrecht in der Tradition von Immanuel Kant, des Völkerbundes und der UNO-Charta eine Bedeutung behalten soll, nicht in eine rechtliche Prärogative der Großmächte zu transformieren. Mit Schmitts Großraum-Theorem ließe sich auch die militärische Intervention der Sowjetunion in der CSSR vom 2 I. August I968, gestützt auf Breschnews Doktrin der begrenzten Souveränität der Länder im sowjetischen Einflussbereich, rechtfertigen. Wenn Großmachtinteressen und internationales Recht unterscheidungslos fusioniert werden, bleibt das Völkerrecht auf der Strecke.

Eine Analyse der NS-Doktrin gelingt offenbar nur dann in vollem Maße, wenn - wie bei Ernst Fraenkel, der sein Buch seiner Frau »in Erinnerung an die gemeinsam erlebten schweren Jahre der bürokratisierten Rechtlosigkeit «24 widmete - die existentielle Distanz zum System planmäßiger Willkür sich mit rechtsstaatlichen Kategorien der Analyse verbindet.

I7 Ulrich Herbert, Best. Biographische Studien über Radikalismus, Weltanschauung und Vernunft, I903i 989 , Bonn I996, S. 249.

I 8 Ernst-Rudolf Huber, Verfassungsrecht des großdeutschen Reiches, Hamburg 1937/39, S. 82 ff.

I9 Carl Schmitt, Positionen und Begriffe im Kampf mit Weimar-Genf-Versailles (1940), Berlin I995, S. 335 ff., $344 \mathrm{ff}$.

20 Dreier III, S. 67, 69.

2 I Dreier III, S. 69.

22 Schmitt (Fn. I 8), S. 342 f.

23 Schmitt (Fn. I 8).

24 Fraenkel (Fn. I). 